\title{
Interfacing Telecom Fibers and Silicon Core Fibers with Nano-Spikes for In-Fiber Silicon Devices
}

\author{
O. Aktas, H. Ren, A. F. J. Runge, A. C. Peacock \\ Optoelectronics Research Centre, University of Southampton, Highfield, Southampton, SO17 1BJ, UK \\ Tel.: (44) 739358 6490,E-mail:O.Aktas@soton.ac.uk \\ T. Hawkins, J. Ballato \\ Center for Optical Materials Science and Engineering Technologies (COMSET), Clemson University, Clemson, SC 29634, USA \\ Department of Materials Science and Engineering, Clemson University, Clemson, SC 29634, USA \\ U. J. Gibson \\ Department of Physics, Norwegian University of Science and Technology, 7491 Trondheim, Norway \\ Applied Physics Department, KTH Royal Institute of Technology, SE-10691 Stockholm, Sweden
}

\begin{abstract}
We report fabrication of tapered silicon core fibers with nano-spikes enabling efficient optical coupling into the core, as well as their seamless integration with single mode fibers. A proof-of-concept integrated in-fiber silicon device is demonstrated.

OCIS codes: (060.1810) Buffers, couplers, routers, switches, and multiplexers; (060.2280) Fiber design and fabrication.
\end{abstract}

\section{Introduction}

In the past decade, there has been significant progress in the production of silicon core optical fibers (SCFs) [1] and two fabrication methods have been developed: (i) molten core fibre drawing [2], and (ii) high pressure assisted chemical deposition (HPCVD) inside silica capillaries [3]. Of these, the former is the more globally practiced and basically depends on conventional fiber drawing of a molten silicon core inside a silica tube. A key advantage of this method is that it is capable of producing many meters of SCFs with continuous polycrystalline silicon cores. Investigations of the as-drawn SCFs have revealed that material absorption due to impurities, scattering from grain boundaries and density fluctuations in the core are the major contributions of optical losses [4]. However, several approaches have been successfully employed to improve the material quality of the as-drawn SCFs, including thermal annealing, laser recrystallization [5], and tapering [6]. So far, propagation losses as low as $1 \mathrm{~dB} / \mathrm{cm}$ have been reported. Photonic and optoelectronic applications such as all-optical modulation based on two-photon absorption [7], and high-speed photodetection [8] have been demonstrated.

Currently, the main challenge hindering the technological exploitations of SCFs, is how to efficiently couple light into the micron-sized silicon cores when interfaced directly with conventional telecom fibers for in-fiber silicon devices. In this regard, we report here the fabrication of tapered SCFs with nano-spikes and show their robust integration with single mode fibers (SMF) [9]. As a proof-of-concept, we demonstrate a $30 \mu \mathrm{m}$ clad diameter SMFSCF device with nano-spike coupling loss of $3.7 \mathrm{~dB}$ and propagation loss of $1.66 \mathrm{~dB} / \mathrm{cm}$. Finite-element-based simulations show that the coupling losses can be reduced below $0.5 \mathrm{~dB}$ if the clad diameters are below $10 \mu \mathrm{m}$.

\section{Fabrication and integration}

The SCFs used in this work were fabricated using the molten core fiber drawing technique. A thin layer of calcium oxide $(\mathrm{CaO})$ was first coated inside the silica capillary tube to prevent oxygen in-diffusing during the process. This coating, which is a mixture of $\mathrm{CaO}$ and $\mathrm{SiO}_{2}$ [10], also serves as an intermediate index cladding (IIC) and stressreducing layer. A phosphorus doped silicon rod $(0.13-0.145 \mathrm{ohm}-\mathrm{cm})$ was then sleeved into the silica tube and was drawn into a fiber using standard fiber drawing process at a temperature of $1950^{\circ} \mathrm{C}$.
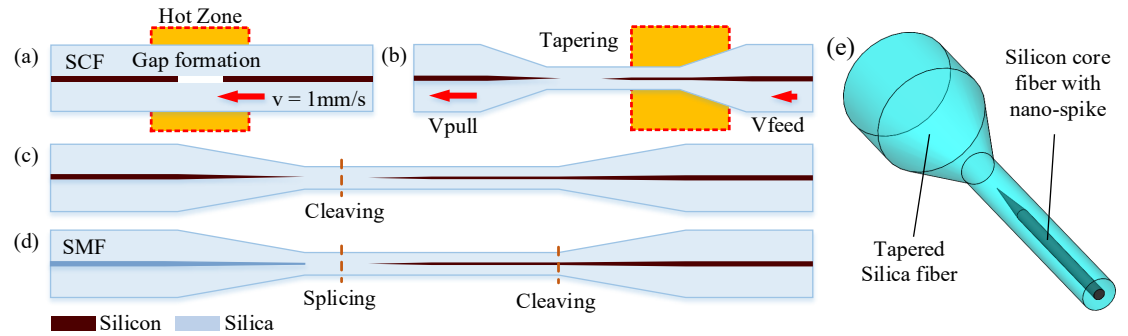

Fig. 1. Steps of fabrication. (a) Void gap formation in the core of an as-drawn SCF after heat-polishing process. (b) Tapering of the void gap in the core results in a silicon-free region with a $\mathrm{CaO} / \mathrm{SiO}_{2}$ core, and a silicon nano-spike. (c) Cleaving of the tapered SCF. (d) Splicing the SCF with a tapered SMF and final cleaving at the other end. (e) An integrated SMF-SCF fiber device with a nano-spike input which enables efficient optical coupling into the silicon core via spliced SMF. 


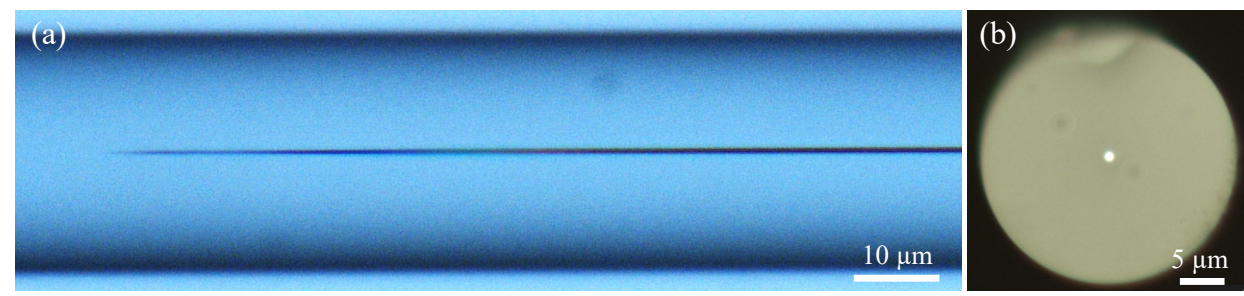

Fig. 2. Optical microscopy images show (a) a tapered silicon core fiber with a nano-spike and (b) the cleaved end facet of the silicon core fiber for output optical coupling.

As-drawn SCFs are poly-crystalline, and have high tensile stress due to the large difference in the thermal expansion coefficients of silica and silicon. This tensile stress can be used to create a void gap by melting the core locally with a heat-polishing method based on unidirectional movement of the as-drawn fiber in a hot zone, as shown schematically in Fig. 1(a). Tapering of the fiber with the void gap is performed by slowly feeding the fiber into the hot zone and pulling it from the other end with a higher pulling speed (see Fig. 1(b)). Collapsing of the void gap results in a silicon-free region with a $\mathrm{CaO} / \mathrm{SiO}_{2}$ core, and a silicon nano-spike at the right end. After cleaving the tapered SCF at the silicon-free region, a tapered SMF can be spliced to this facet, as shown in Figs. 1(c) and 1(d). An integrated fiber device (see Fig. 1(e)) is finally obtained releasing the tapered SCF after cleaving.

We used a Vytran GPX-3400 glass processing system to taper the SCFs. Local melting of the silicon core before tapering was used to create a $150 \mu \mathrm{m}$ long void in the core. Subsequent tapering of the fiber with $1 \mathrm{~mm} / \mathrm{s} \mathrm{pulling}$ speed and $68 \mathrm{~W}$ power for the heater resulted in a long silicon-free fiber section and a silicon core with a nano-spike due to the collapse of the softened silica cladding, as shown in Fig. 2(a). The cleaved end facet of the fiber, which is used for free space coupling, is also shown in Fig. 2(b). Cladding and core diameters of the tapered SCF are $30 \mu \mathrm{m}$ and $1.1 \mu \mathrm{m}$, respectively. Later, we adiabatically tapered an SMF fiber to obtain a waist region with the same outer diameter as the silicon fiber and cleaved it in the waist region for splicing to the silicon-free part of the tapered SCF. A silica region between the splicing point and nano-spike assures that the silicon nano-spike is not affected by the high temperature applied during the splicing. Another advantage is splicing occurs only at silica-silica interface, which is not affected by Fresnel reflection loss.

\section{Simulations}

FEM-based simulations were used to calculate optical coupling losses of the nano-spike for different cladding diameters $(D=10-30 \mu \mathrm{m})$ and IIC layer thicknesses $(h=0-400 \mathrm{~nm})$ at $\lambda=1550 \mathrm{~nm}$, using COMSOL software. The nano-spike starts from $5 \mathrm{~nm}$ in diameter and widens to a core diameter of $1 \mu \mathrm{m}$ at the end of the transition length of $200 \mu \mathrm{m}$. A nano-spike encapsulating layer (IIC) with a refractive index of 1.6 was used to calculate coupling losses from the fundamental mode of a $\mathrm{CaO} / \mathrm{SiO}_{2}$ core $(\mathrm{n}=1.6, \mathrm{r}=\mathrm{h})$ to the fundamental mode of a SCF. The spike coupling loss was calculated to be $0.35 \mathrm{~dB}$ for a SCF with a cladding diameter of $10 \mu \mathrm{m}$ and a core diameter of 1 $\mu \mathrm{m}$. The profile of the electric field strength through the fiber is shown in Fig. 3(a) for these parameters. Presence of the IIC layer improves optical coupling to the high index $(\mathrm{n}=3.48)$ silicon nano-taper, as shown in Fig. 3(b). For our SMF-SCF device paramters, the nano-spike coupling loss and the mode conversion loss at the splice point are calculated to be $3.9 \mathrm{~dB}$ and $1.25 \mathrm{~dB}$, respectively.

(a)

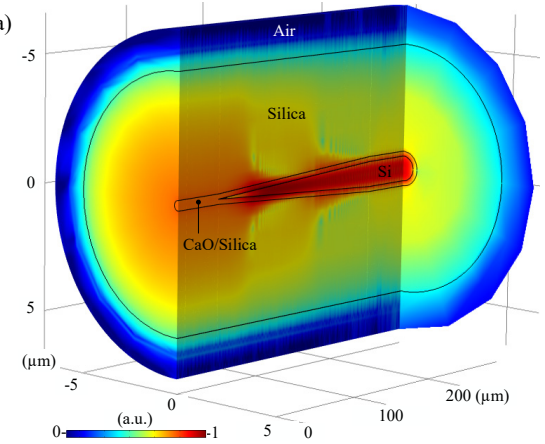

(b)

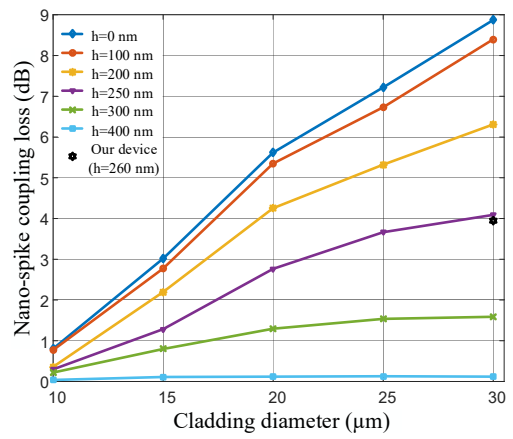

Fig. 3. (a) Simulation of light coupling into a silicon core via the nano-spike encapsulated with an intermediate index (IIC) cladding layer of $200 \mathrm{~nm}$ thickness and a silica cladding of $10 \mu \mathrm{m}$ diameter. Spike coupling loss is $0.35 \mathrm{~dB}$ for this geometry. Electric field strength is shown in normalized logarithmic scale. (b) Spike coupling loss versus cladding diameter for various IIC layer thicknesses $\mathrm{h}$. Calculated nano-spike coupling loss for our device parameters is designated with a star. 


\section{Optical Characterization}

The SMF-SCF device under test was connected to an infrared continuous-wave (CW) laser (Tunics T100S-HP) with a fiber coupled output of $1 \mathrm{~mW}$ at $\lambda=1550 \mathrm{~nm}$ and a $63 \mathrm{x}$ objective lens with a NA of 0.85 to collect the output power from the cleaved facet. The output mode is a superposition of cladding guided and core guided modes. The images taken by a beam profiler (Spiricon) before and after spatial filtering are shown in Figs. 4(a) and 4(b), respectively. The power was measured at 5 different cut-back positions along the device as shown in Fig. 4(c).

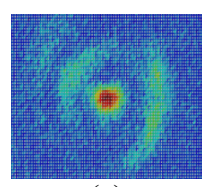

(a)

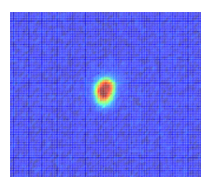

(b)

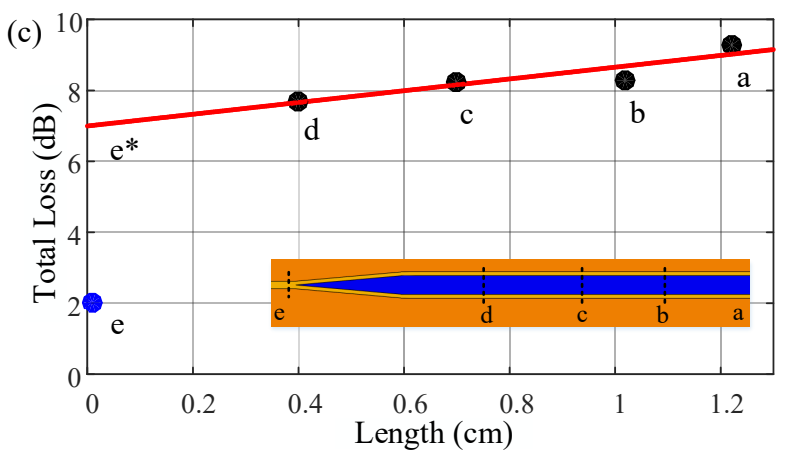

Fig. 4. (a) Experimental output mode profile image taken by a beam profiler. (b) Output mode profile after spatial filtering. (c) Total loss measurements with a linear fit to the data. Inset shows the cut-back positions.

Initially, the total transmitted power was $330 \mu \mathrm{W}$. After spatial filtering, the power of the core guided mode was measured to be $118 \mu \mathrm{W}$ at position $a$, which corresponds to a total loss of $9.2 \mathrm{~dB}$. A linear fit to the cut-back data was used to calculate a propagation loss of $1.66 \pm 0.54 \mathrm{~dB} / \mathrm{cm}$. After the nano-spike was removed at position $e$, a loss of $2 \mathrm{~dB}$ was measured, which includes fiber connector loss, SMF tapering loss, and the mode conversion loss at the splicing point. The estimated loss of the device $(7 \mathrm{~dB})$ at the nano-spike input was determined from the $\mathrm{y}$ intercept of the linear fit. The difference between this estimated loss at position $e^{*}$ and the measured loss at position $e$ gives a total coupling loss of $\sim 5 \mathrm{~dB}$ at the nano-spike, which includes the nano-spike coupling loss as well as an extra loss due to the change of interface from Si-air $(\sim 1.5 \mathrm{~dB})$ to $\mathrm{CaO} / \mathrm{SiO}_{2}$-air $(\sim 0.2 \mathrm{~dB})$ at the position $e$. Accounting for this extra loss, the nano-spike coupling loss was estimated to be $\sim 3.7 \mathrm{~dB}$, which is in accordance with the simulated value of $3.9 \mathrm{~dB}$. The nano-spike coupling losses could be reduced to below $0.5 \mathrm{~dB}$ if the cladding diameters are decreased to $10 \mu \mathrm{m}$, as shown by the simulations in Fig. 3(b).

\section{Conclusion}

Reported here was the fabrication of tapered SCFs with nano-spikes and their robust integration with SMFs by splicing. Loss measurements have revealed the propagation and nano-spike coupling losses for our $30 \mu \mathrm{m}$ cladding diameter device to be below $2 \mathrm{~dB} / \mathrm{cm}$ and $4 \mathrm{~dB}$, respectively. Seamless integration of SMFs and SCFs can pave the way for in-fiber silicon devices such as SCF-based Raman amplifiers and all-fiber photodetectors.

\section{References}

[1] A. C. Peacock, U. J. Gibson, and J. Ballato, "Silicon optical fibres - past, present, and future," Adv. Phys.:X 1, 114-127 (2016).

[2] J. Ballato, T. Hawkins, P. Foy, R. Stolen, B. Kokuoz, M. Ellison, C. McMillen, J. Reppert, A. M. Rao, M. Daw, S. Sharma, R. Shori, O. Stafsudd, R. R. Rice, and D. R. Powers, “Silicon optical fiber," Opt. Express 16, 18675-18683 (2008).

[3] P. J. A. Sazio, A. Amezcua-Correa, C. E. Finlayson, J. R. Hayes, T. J. Scheidemantel, N. F. Baril, B. R. Jackson, D.-J. Won, F. Zhang, E. R. Margine, V. Gopalan, V. H. Crespi, and J. V. Badding, "Microstructured optical fibers as high-pressure microfluidic reactors," Science 311, 1583-1586 (2006)

[4] S. Morris, T. Hawkins, P. Foy, J. Hudson, L. Zhu, R. Stolen, R. Rice, and J. Ballato, "On loss in silicon core optical fibers," Opt. Mater. Express 2, 174510 (2012).

[5] N. Healy, M. Fokine, Y. Franz, T. Hawkins, M. Jones, J. Ballato, A. C. Peacock, and U. J. Gibson, " $\mathrm{CO}_{2}$ laser-induced directional Recrystallization to produce single crystal silicon-core optical fibers with low loss," Adv. Opt. Mater. 4, 1004-1008 (2016).

[6] Y. Franz, A. F. J. Runge, H. Ren, N. Healy, K. Ignatyev, M. Jones, T. Hawkins, J. Ballato, U. J. Gibson, and A. C. Peacock, "Material properties of tapered crystalline silicon core fibers," Opt. Mater. Express 7, 2055-2061 (2017).

[7] P. Mehta, N. Healy, T. D. Day, J. R. Sparks, P. J. A. Sazio, J. V. Badding, and A. C. Peacock, "All-optical modulation using two-photon absorption in silicon core optical fibers," Opt. Express 19, 19078-19083 (2011).

[8] R. He, T. D. Day, M. Krishnamurthi, J. R. Sparks, P. J. A. Sazio, V. Gopalan , and J. V. Badding, "Silicon p-i-n Junction Fibers," Adv. Mater. 25, 1461-1467 (2013).

[9] H. Ren, O. Aktas, Y. Franz, A. F. J. Runge, T. Hawkins, J. Ballato, U. J. Gibson, and A. C. Peacock, "Tapered silicon core fibers with nanospikes for optical coupling via spliced silica fibers," Opt. Express 25, 24157-24263 (2017).

[10] E. F. Nordstrand, A. N. Dibbs, A. J. Eraker, and U. J. Gibson, "Alkaline oxide interface modifiers for silicon fiber production," Opt. Mater. Express 3, 651-657 (2013). 